\section{SCIENCE CHINA \\ Physics, Mechanics \& Astronomy}

-News \& Views•
April 2021 Vol. 64 No. 4: 244305

https://doi.org/10.1007/s11433-020-1643-x

\title{
Quadratic Weyl points in metacrystals
}

\author{
ChunYin Qiu*
}

School of Physics and Technology, Wuhan University, Wuhan 430072, China

Received October 16, 2020; accepted November 23, 2020; published online January 28, 2021

Citation: C. Y. Qiu, Quadratic Weyl points in metacrystals, Sci. China-Phys. Mech. Astron. 64, 244305 (2021), https://doi.org/10.1007/s11433-020-1643-x

The discovery of new topological states has become an important goal in the multidisciplinary fields of physics and material science [1,2]. In addition to topological insulators, topological semimetals without band gaps have been the focus of research efforts. Typical examples include Weyl and Dirac semimetals, which feature isolated 2-band and 4-band linear crossing points in their band structures. Intriguingly, such linearly dispersive quasiparticles in solids can mimic the behavior of high-energy particles (e.g., Dirac and Weyl fermions in the relativistic region); therefore, these materials provide easily accessible, low-energy platforms to investigate the properties of their high-energy counterparts. Recently, novel quasiparticles beyond conventional highenergy particles have garnered tremendous attention $[3,4]$. Herein, I briefly introduce two independent experimental studies on unconventional Weyl quasiparticles. Unlike conventional Weyl points (CWPs), which are linearly crossed with two bands along all directions (Figure 1(a)), the unconventional Weyl points exhibit dispersions quadratic in two directions and linear in the third one (Figure 1(b)).

In general, unconventional quasiparticles correspond to multifold band crossings and host topological monopole charges higher than $1[3,4]$. Among these quasiparticles, the so-called spin-1 Weyl points and charge-2 Dirac points have been experimentally observed $[5,6]$ immediately after their predictions. However, unlike the CWPs crossed with two bands (Figure 1(a)), the spin-1 Weyl points are constructed with a linear Weyl cone plus one additional flat band (Figure 1(c)), and charge-2 Dirac points comprise two CWPs

*Corresponding author (email: cyqiu@whu.edu.cn) of identical monopole charges (Figure 1(d)). Both of these newly observed unconventional quasiparticles host nontrivial monopole charges of \pm 2 , which are protected by the crystalline symmetries of chiral crystals and occur simultaneously at different high-symmetry momenta of the same chiral crystal [3-6]. Furthermore, unconventional quasiparticles can be simply formed through two-band crossings, around which the dispersions are quadratic in two directions and linear in the third one (Figure 1(b)), which is remarkably different from the aforementioned multiband systems. Such two-band and quadratically dispersive topological nodes, called quadratic Weyl points (QWPs), host a chiral charge of 2 as well. However, despite predicted earlier than those multiband high charge quasiparticles, such QWPs have not been observed owing to a shortage of ideal candidates in real materials.

Metacrystals have been recently demonstrated to be exceptional platforms for constructing and identifying new topological phases. The systems, macroscopic in their physical dimensions, are thermally and chemically stable and are easy to fabricate with desired spatial symmetries through the arrangement of appropriately-shaped artificial atoms in specific lattices. In particular, the macroscopic features of the artificial crystals enable flexible engineering of the surface structures (allowing the tailoring of topological surface states) and direct visualization of exceptional surface phenomena such as topological negative refractions $[7,8]$. Although the desirable QWPs have been theoretically demonstrated in electromagnetic and acoustic metacrystals, there is no compelling experimental evidence offered for their existence. 
(a)

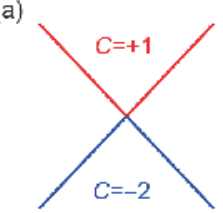

Conventional Weyl

(e)

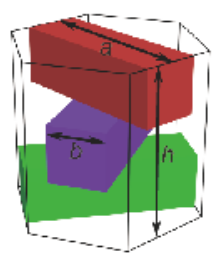

(b)

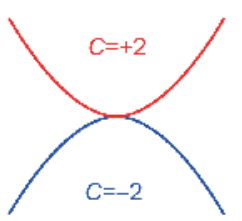

Quadratic Weyl

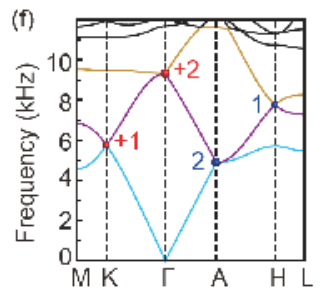

(c)

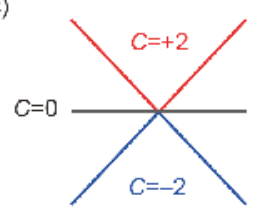

Spin-1 Weyl

(g)

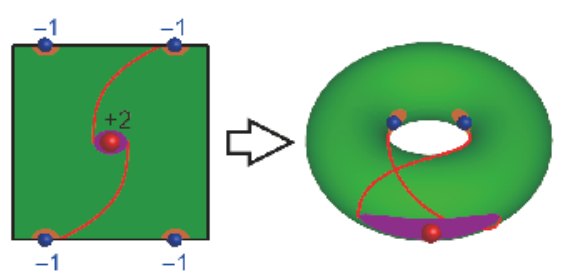

(d)

Charge-2 Dirac

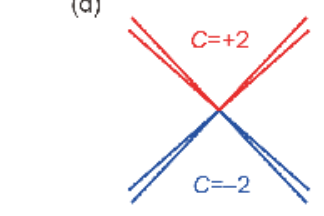

.

Figure 1 (Color online) Conventional and unconventional quasiparticles. (a) A conventional Weyl point (CWP) crossed with two bands linearly along all directions. (b) A quadratic Weyl point (QWP) formed by two bands quadratically along two directions and linearly along the third direction. (c) A spin-1 Weyl point formed by two linear bands plus one extra flat band. (d) A charge-2 Dirac point overlapped with two CWPs of the same charges. Chern numbers are labeled for the corresponding energy bands. (e) Unit cell geometry of the acoustic metacrystal used in ref. [9]. (f) Band structure of the acoustic metacrystal. (g) Schematic illustration of the surface arc connectivity between the charge-2 QWP and charge-1 CWPs.

Recently, He et al. [9] presented the first experimental observation of the QWPs based on a three dimensional (3D) acoustic metacrystal. Figure 1(e) shows the geometry of the unit cell, which is stacked with identical square acrylic rods at an anticlockwise twist of $2 \pi / 3$ along the $z$ direction. Airborne sound is considered the remaining volume of the structure. The resulting 3D acoustic metacrystal exhibits a chiral structure that lacks mirror and inversion symmetry. Owing to the threefold screw symmetry, the band structure (Figure 1(f)) of the system hosts both CWPs and QWPs at different high-symmetry momenta. The widely separated topological nodal points result in giant surface arcs that greatly benefit their experimental detection. In particular, the topological surface states exhibit unique global connectivity in frequency-momentum space. As illustrated in Figure 1(g), the isofrequency contour features one pair of time-reversalrelated open arcs connecting the QWP and two CWPs, which is noncontractible because of the symmetry-enforced highsymmetry momenta of the QWP and CWPs. Based on the macroscopic experiments, He et al. [9] have not only confirmed the unusual quadratic dispersions around the QWPs through the measurement of the projected bulk bands but also identified the double-helicoid surface arcs through Fourier transforming the scanned surface fields.

A similar study was later presented by Yang et al. [10] using a 3D metacrystal working for microwaves. In addition to observing long surface arcs spanning a wide frequency window, they demonstrate that the surface states exhibit a novel topological self-collimation property and are robust against disorders.

In summary, metacrystals of classical waves have been proven to be an excellent experimental platform to investigate the intriguing physical phenomena associated with QWPs. These findings will significantly influence both fundamental physics and material science and will receive considerable interest among the physics community, ranging from condensed matter physics to photonics and acoustics.

1 M. Z. Hasan, and C. L. Kane, Rev. Mod. Phys. 82, 3045 (2010), arXiv: 1002.3895 .

2 X. L. Qi, and S. C. Zhang, Rev. Mod. Phys. 83, 1057 (2011), arXiv: 1008.2026.

3 B. Bradlyn, J. Cano, Z. Wang, M. G. Vergniory, C. Felser, R. J. Cava, and B. A. Bernevig, Science 353, aaf5037 (2016).

4 C. Fang, M. J. Gilbert, X. Dai, and B. A. Bernevig, Phys. Rev. Lett. 108, 266802 (2012), arXiv: 1111.7309.

5 Z. Rao, H. Li, T. Zhang, S. Tian, C. Li, B. Fu, C. Tang, L. Wang, Z. Li, W. Fan, J. Li, Y. Huang, Z. Liu, Y. Long, C. Fang, H. Weng, Y. Shi, H. Lei, Y. Sun, T. Qian, and H. Ding, Nature 567, 496 (2019).

6 D. S. Sanchez, I. Belopolski, T. A. Cochran, X. Xu, J. X. Yin, G. Chang, W. Xie, K. Manna, V. Süß, C. Y. Huang, N. Alidoust, D. Multer, S. S. Zhang, N. Shumiya, X. Wang, G. Q. Wang, T. R. Chang, C. Felser, S. Y. Xu, S. Jia, H. Lin, and M. Z. Hasan, Nature 567, 500 (2019).

7 H. He, C. Qiu, L. Ye, X. Cai, X. Fan, M. Ke, F. Zhang, and Z. Liu, Nature 560, 61 (2018), arXiv: 1808.04647.

8 Y. Yang, H. Sun, J. Xia, H. Xue, Z. Gao, Y. Ge, D. Jia, S. Yuan, Y. Chong, and B. Zhang, Nat. Phys. 15, 645 (2019), arXiv: 1811.06735.

9 H. He, C. Qiu, X. Cai, M. Xiao, M. Ke, F. Zhang, and Z. Liu, Nat. Commun. 11, 1820 (2020), arXiv: 1911.00806.

10 Y. Yang, Z. Gao, X. Feng, Y. X. Huang, P. Zhou, S. A. Yang, Y. Chong, and B. Zhang, Phys. Rev. Lett. 125, 143001 (2020), arXiv: 2007.02003. 